\title{
Thermal and Infrared Spectroscopic Studies on Hydrogen-Bonding Interaction between Poly(3-hydroxybutyrate) and Catechin
}

\author{
Jianchun Li, Bo ZHU, Yong HE, and Yoshio INOUE ${ }^{\dagger}$ \\ Department of Biomolecular Engineering, Tokyo Institute of Technology, \\ Nagatsuta 4259, Midori-ku, Yokohama 226-8501, Japan
}

(Received November 28, 2002; Accepted February 19, 2003)

\begin{abstract}
The effects of catechin on the properties of bacterially synthesized isotactic poly $[(R)-3-$ hydroxybutyrate] $(i$-PHB) and chemosynthesized highly-syndiotactic poly[ $(R, S)$-3-hydroxybutyrate $](s$-PHB) were investigated by differential scanning calorimetry and Fourier transform infrared (FT-IR). In both PHB/catechin blend systems, the composition-dependent single glass transition temperature was observed. By using FT-IR, the formation of intermolecular hydrogen bonds was found between the carbonyl of PHB and the hydroxyl of catechin. The strength of intermolecular hydrogen bonds of $s$-PHB/catechin blends, $i$-PHB /catechin blends and pure catechin is in this order, i.e., $s$-PHB/catechin blends $<i$-PHB/catechin blends $<$ pure catechin. The crystallization of either $i$-PHB or $s$-PHB was found to be restrained by the existence of catechin, and both $i$-PHB and $s$-PHB are miscible with catechin in the amorphous phase due to the intermolecular hydrogen-bonding. The effect of different stereochemical structures of $i$-PHB and $s$-PHB on the PHB-catechin interaction has been discussed.
\end{abstract}

KEY WORDS Biodegradable / Blends / Catechin / Hydrogen Bond / Miscibility / Poly(3-hydroxybutyrate) /

Bacterially synthesized poly $[(R)$-3-hydroxybutyrate $]$ $(i$-PHB) is an environmentally biodegradable and biocompatible thermoplastic material with the physical properties similar to those of traditional plastics. However, its wider applications have been prevented by some obstacles such as poor mechanical property induced by its big spherulites and high extent of secondary crystallization. Some methods are used to modify its thermal and mechanical properties. Especially for the method of blending, if the intermolecular hydrogen bonds between two components are introduced, usually the miscibility of the blends is increased to improve the thermal and mechanical properties of the materials.

It has become clear that phenolic polymer such as poly ( $p$-vinyl phenol) $(\mathrm{PVPh})$ and 4,4'-thiodiphenol (TDP) possess excellent potential for hydrogenbonding interactions with proton-acceptor polymers, ${ }^{1-7}$ since the hydroxyl group in the $p$-position of the phenyl ring of the repeat unit is easily accessible. All these researches help a better understanding of the relationship between the formation of hydrogen bonds and the chemical structures of two partners. However, the phenolic compounds, as proton-donating components used in the study of hydrogen-bonding interaction, are mainly chemosynthesized and even toxic to human body. Using naturally produced compounds instead of chemosynthesized compounds to modify other polymers in industry has been encouraged due to the emerging regulatory requirement of sustainable development. So far as we know, there are almost no studies in naturally produced phenolic compound and proton-acceptor polymer blend system based on the intermolecular hydrogen-bonding. As an attempt, a naturally produced phenolic compound, green tea catechin (its chemical structure is shown in Chart 1), is selected as the candidate to substitute chemosynthesized phenolic compounds like TDP. Actually, phenolic compounds are the secondary plant metabolites found in all fruits and vegetables, including apples, coffee beans, grapes, prunes, and tea leaves. ${ }^{9}$ Catechin itself is the main component of phenolic compound in tea

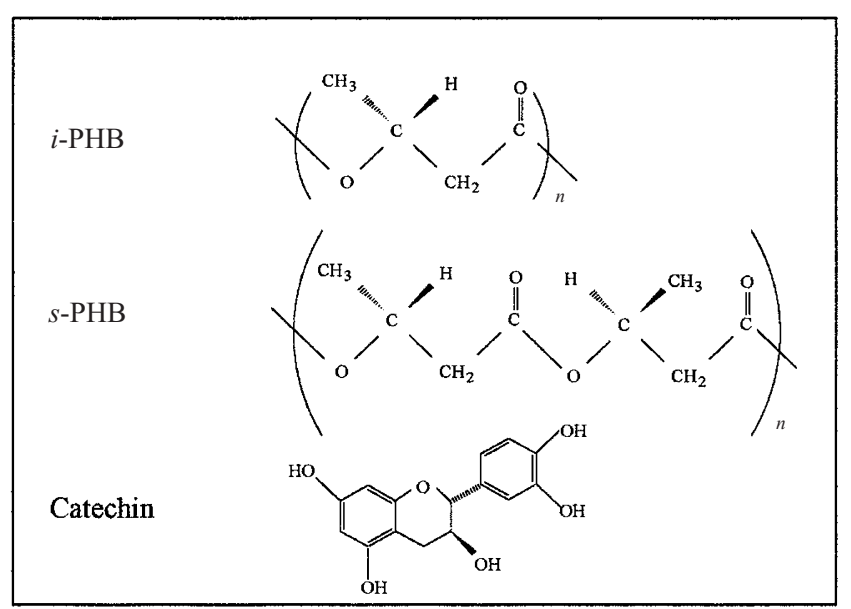

Chart 1. The chemical structures of $i$-PHB, $s$-PHB, and catechin.

${ }^{\dagger}$ To whom correspondence should be addressed (Tel: +81-45-924-5794, Fax: +81-45-924-5827, E-mail: yinoue@bio.titech.ac.jp). 
leaves and possesses outstanding antioxidant and free radical-scavenging properties. ${ }^{10,11}$

On the other hand, the study on the hydrogenbonding of biocompatible phenolic compounds is so important to the controlled drug delivery technology since phenolic groups widely exist in biological and biomedical materials. Furthermore, the protonacceptor polymers like polyesters or polyethers are widely used as biomedical materials such as carriers in drug delivery systems. It is thought that controlled drug delivery technology represents one of the most rapidly advancing areas of science contributing to human health care. ${ }^{12}$ Although both biodegradable and non-biodegradable polymers have been used as carriers in drug delivery systems, biodegradable systems have gained much of the recent attention and development because non-biodegradable systems need retrieval or further manipulation after introduction into the human body. Currently biodegradable polymers at least have two major applications: one is as biomedical polymers that contribute to the medical care of patients and the other is as ecological polymers that keep the earth environments clean. ${ }^{13}$ The study on the intermolecular interaction between PHB and phenolic compound seems important because PHB is thought as a promising candidate in controlled drug delivery technology and phenolic compound widely exists in drugs. Both $i$-PHB and chemosynthesized highly-syndiotactic poly[ $(R, S)$-3-hydroxybutyrate] ( $s$-PHB) are reported to be biodegradable. ${ }^{14}$ The weak self-association of these two polymers makes them good components to study the interaction between the carbonyl group of polyester and the hydroxyl group of polyphenol. $i$ - And $s$-PHB share the same chemical structure but different stereochemical structures as shown in Chart 1 , which afford them different physical properties. $i$-PHB is a highly crystalline polymer with the crystallinity more than $50 \%,{ }^{15}$ while $s$-PHB is crystalline but almost an amorphous polymer. ${ }^{16}$

In this light, the purpose of this study is to evaluate the hydrogen-bonding interaction between naturally produced phenolic compound catechin and protonacceptor polymer PHB, and then the modification of PHB by hydrogen-bonding between the two components would been emphasized. The processability of PHB modified by the addition of catechin is expected. The miscibility and thermal properties of $i$-PHB and $s$-PHB/catechin blends will be investigated by differential scanning calorimetry (DSC). The specific interaction between PHB and catechin will be studied by Fourier transform infrared spectroscopy (FT-IR).

\section{EXPERIMENTAL}

\section{Materials}

Biosynthesized isotactic poly $[(R)-3$-hydroxybutyrate] $\left(i-\mathrm{PHB} ; M_{\mathrm{w}}=5.86 \times 10^{5}, M_{\mathrm{w}} / M_{\mathrm{n}}=2.35\right)$ was supplied by the courtesy of Mitsubishi Gas Co. Ltd., Japan. Chemosynthesized highly-syndiotactic poly[(R,S)-3-hydroxybutyrate] ( $s$-PHB; diad syndiotacticity is $\left.0.6 ; M_{\mathrm{w}}=9.20 \times 10^{4}, M_{\mathrm{w}} / M_{\mathrm{n}}=1.64\right)^{17}$ was supplied by the courtesy of Takasago International Corporation, Japan. Catechin (C-1251, Lot No. 120K1194) was purchased from Aldrich Chemical Co., Ltd. The solvents were used as received.

\section{Preparation of Blend Samples}

The blend films of $i$-PHB/catechin were prepared by casting the mixture of solution of $i$-PHB in chloroform $(5 \mathrm{wt} \%)$ and catechin in dioxane $(5 \mathrm{wt} \%)$ to the Teflon petri dish, allowing the solvents to evaporate at room temperature, while the blend films of $s$-PHB/catechin were prepared by casting the $5 \mathrm{wt} \%$ MEK solution of both solutes. Furthermore, all the blend films were kept in vacuum at $60^{\circ} \mathrm{C}$ for one day to remove the residual solvent and then kept in vacuum more than two weeks at room temperature.

\section{Differential Scanning Calorimetry (DSC)}

DSC analysis was performed on a SEIKO DSC 220 system (Seiko Instrument, Co., Tokyo, Japan). The polymer sample $(c a .5 \mathrm{mg})$ packed in an aluminum pan was heated from -50 to $200^{\circ} \mathrm{C}$ at a heating rate of $20^{\circ} \mathrm{C} \mathrm{min}^{-1}$. Afterwards, the sample was rapidly quenched by liquid nitrogen to $-60^{\circ} \mathrm{C}$, and reheated from -50 to $200^{\circ} \mathrm{C}$ also at a scanning rate of $20^{\circ} \mathrm{C} \mathrm{min}^{-1}$.

Melting point $T_{\mathrm{m}}$ was taken as the endothermic peak top in the thermal diagram recorded by the first heating scan, and the heat of fusion $\Delta H$ was calculated from the integral of the endothermic melting peak in DSC curve. Glass transition temperature $T_{\mathrm{g}}$ was taken as the midpoint of the glass transition recorded in the second heating scan.

\section{Fourier Transform Infrared (FT-IR) Spectroscopy}

The thin layers of the $i$-PHB/catechin and $s$ $\mathrm{PHB} /$ catechin blends with a suitable thickness for FTIR measurements were prepared by casting the blend solution ( $c a .20 \mathrm{mg} \mathrm{mL}^{-1}$ ) on the surface of silicon wafers. The maximum absorption of the samples was lower than 1 absorbance unit to ensure the absorption within the linearity range of the detector. The samples were placed for one day at $60^{\circ} \mathrm{C}$ under vacuum 
to eliminate the solvent completely. IR measurements were carried out on a single beam IR spectrometer (PerkinElmer Spectra 2000) at room temperature or at $190^{\circ} \mathrm{C}$ under $\mathrm{N}_{2}$ purging. All the spectra were recorded from 600 to $4000 \mathrm{~cm}^{-1}$ at a resolution of $4 \mathrm{~cm}^{-1}$ with an accumulation of 64 scans.

\section{Line-Shape Analysis of FT-IR Spectra}

A curve-fitting program, based on the Gauss-Newton iteration procedure, ${ }^{18}$ was applied here for the lineshape analysis of the FT-IR carbonyl-vibration band. With the least-squares parameter-adjustment criterion, the band of carbonyl-vibration could be quantitatively resolved into three parts: the amorphous, the crystalline, and the hydrogen-bonded components. This fitting adjusted the peak position, the line shape, peak width and height in such a way that the best fit with the error less than 3\% between experimental and calculated spectra was obtained.

\section{RESULTS AND DISCUSSION}

\section{DSC Analysis of $i-P H B / C a t e c h i n$ and $s-P H B / C a t e c h i n$ Blends}

DSC analysis is usually used to provide some evidence of miscibility of polymer blends according to physical phenomena such as glass transition, melting, and crystallization. In Figure 1 are shown the DSC thermograms of $i$-PHB, catechin and their blends in the first heating scan. The thermogram of $i$-PHB has a strong melting peak at $179.5^{\circ} \mathrm{C}$ and the $T_{\mathrm{g}}$ is not so obvious due to the high content of crystalline phase. Compared to that of $i$-PHB, the thermogram of catechin is much

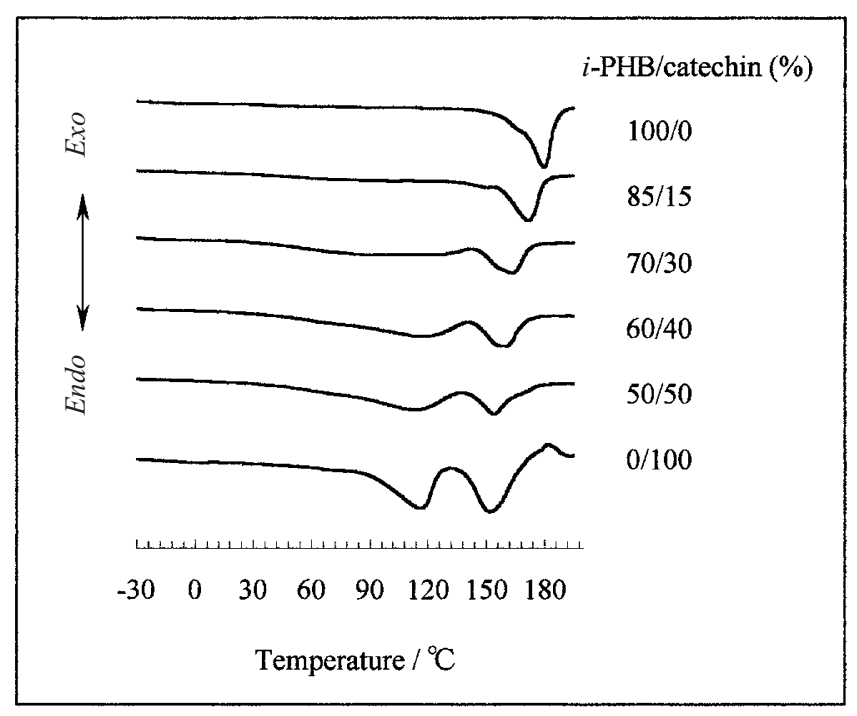

Figure 1. The DSC curves of $i$-PHB and its blends with various contents of catechin recorded during the first heating scan at the heating rate of $20^{\circ} \mathrm{C} \mathrm{min}^{-1}$. complex. It shows two endothermic peaks at $115.9^{\circ} \mathrm{C}$ and $152.4{ }^{\circ} \mathrm{C}$, which are corresponding to the loss of crystal water and the melting of crystalline phase, respectively. What is interesting is that no melting peak corresponding to the catechin component could be observed obviously when the content of catechin in the blends is not more than $30 \%$. This might suggest that the catechin component exists in an amorphous state and its crystallization is completely suppressed in the blends. This phenomenon is very similar to that observed for TDP in the poly(3-hydroxybutyrate-co-4hydroxybutyrate)/TDP blend system. ${ }^{4}$ The crystallinity of $i$-PBH in the blends, $X^{*}$, is estimated from the melting enthalpy, $\Delta H$, in the first scan and the reference melting enthalpy of $100 \%$ crystalline $i$-PHB, $\Delta H_{\text {ref }}$,

$$
X^{*}=\Delta H /\left(W_{i-\mathrm{PHB}} \Delta H_{\mathrm{ref}}\right)
$$

where $\mathrm{W}_{i-\mathrm{PHB}}$ is the weight fraction of $i$-PHB and $\Delta H_{\text {ref }}$ is $146.6 \mathrm{~J} \mathrm{~g}^{-1} .{ }^{19}$ The calculated crystallinity of $i-$ PHB is $51.6 \%$ but that in $i$-PHB/catechin $(70 / 30)$ blend is only $34.7 \%$. While the content of catechin is more than $30 \%, X^{*}$ increases. It can be explained that the melting peak of catechin overlaps that of $i$-PHB and the accurate value of $\Delta H$ of $i$-PHB is not easy to get. Similarly, the accurate $T_{\mathrm{m}}$ of $i$-PHB in the blends cannot be got; however, it is found that the melting peak of $i$-PHB obviously shifts to lower temperature with increasing the catechin content. The melting temperature corresponding to $i$-PHB decreases from $179.5^{\circ} \mathrm{C}$ in the pure state to about $163.9^{\circ} \mathrm{C}$ in $i-\mathrm{PHB} /$ catechin (70/30). It is thought that there is strong intermolecular interaction between $i$-PHB and catechin and the existence of catechin affects the crystallization of $i$-PHB.

In order to eliminate the influence of crystalline phase of $i$-PHB on the glass transition of the blends, the $T_{\mathrm{g}}$ value is measured in the second scan for the blend quenched from the melt state, in which the component of $i$-PHB is almost in the amorphous state. As shown in Figure 2, the $T_{\mathrm{g}}$ value for pure $i$-PHB is $9.3{ }^{\circ} \mathrm{C}$. When $i$-PHB is blended with catechin, the single glass transition is observed and it shifts gradually to higher temperature with increasing catechin content. Usually, the miscibility of phenolic blends depends on both the strength of self-association of the phenolic component and that of inter-association between the phenolic and the other component. It is a simple and useful method to analyze the miscibility of a polymer blend by determining the $T_{\mathrm{g}}$ as a function of the composition. The observation of a single composition-dependent $T_{\mathrm{g}}$ that lies between the $T_{\mathrm{g}}$ values of the pure components is normally taken as the evidence of the blend miscibility with dimensions of about $20-40 \mathrm{~nm}^{20-22}$ However, the low-molecular-weight-molecule catechin is free from 


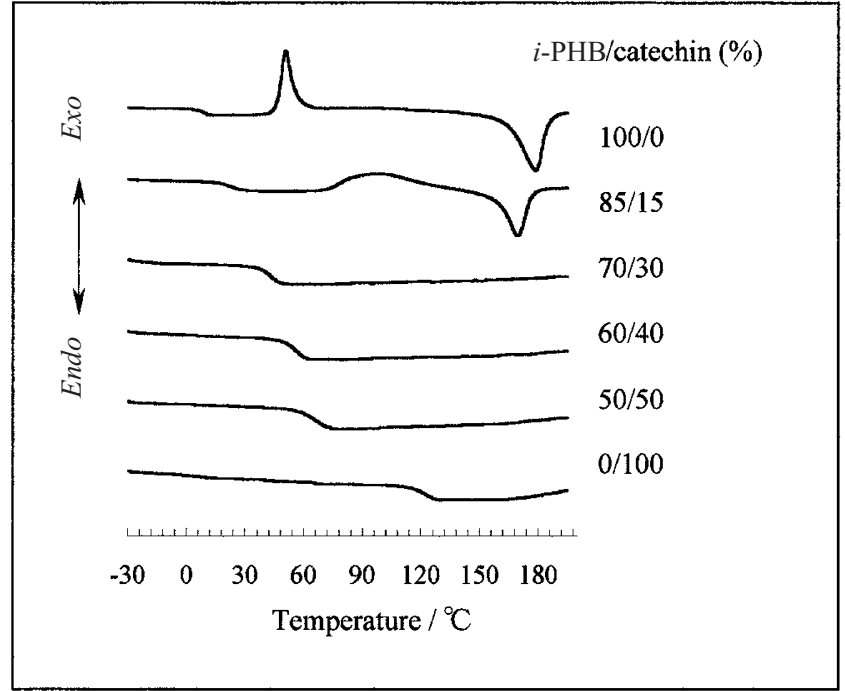

Figure 2. The DSC curves of $i$-PHB and its blends with various contents of catechin recorded during the second heating scan at the heating rate of $20^{\circ} \mathrm{C} \mathrm{min}^{-1}$.

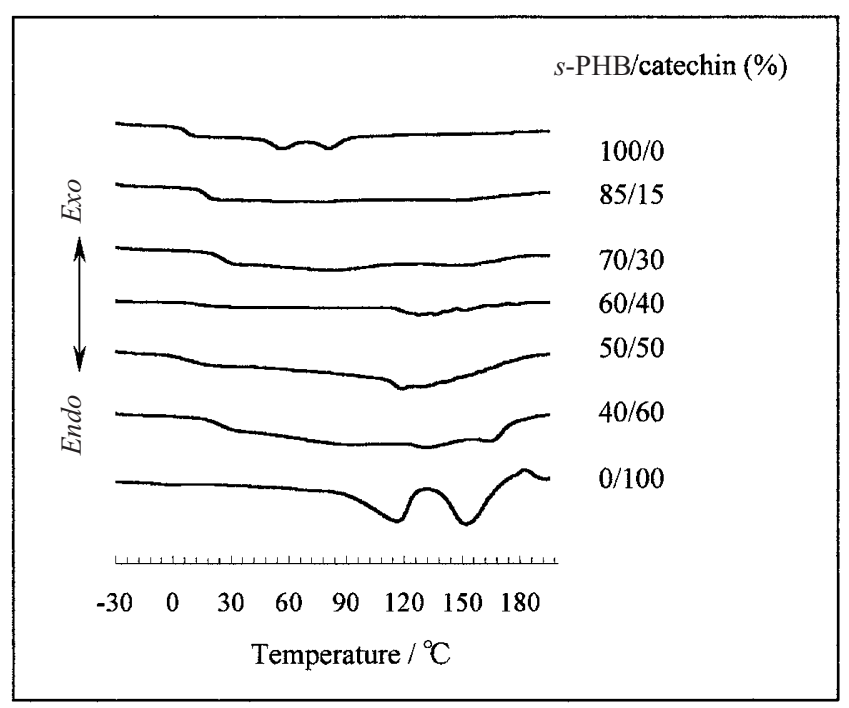

Figure 3. The DSC curves of $s$-PHB and its blends with various contents of catechin recorded during the first heating scan at the heating rate of $20^{\circ} \mathrm{C} \mathrm{min}^{-1}$.

crystallization and shows a glass transition at $123.5^{\circ} \mathrm{C}$. It is thought that the molecules of catechin are highly cross-linked through self-associated hydrogen bonds, which makes assembly of catechin molecules act as a polymer chain-like either in the pure state or in the $i$ PHB/catechin blend system.

In Figure 3, the first heating DSC curve of the chemosynthesized $s$-PHB shows two small melting peaks at $56.1^{\circ} \mathrm{C}$ and $81.3^{\circ} \mathrm{C}$, respectively. ${ }^{16}$ When $s$ PHB is blended with catechin, the melting peaks disappear and $s$-PHB keeps in a fully amorphous state. As shown in Figure 4, $s$-PHB shows a single $T_{\mathrm{g}}$ at $10.5^{\circ} \mathrm{C}$ observed in the second DSC scan, which is very close to that of $i$-PHB. With increasing the content of cate-

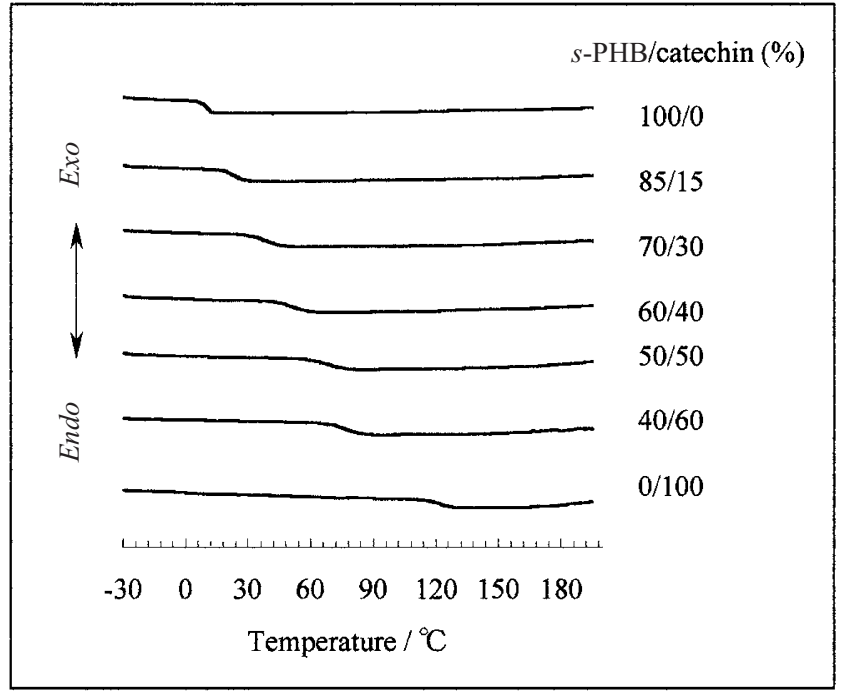

Figure 4. The DSC curves of $s$-PHB and its blends with various contents of catechin recorded during the second heating scan at the heating rate of $20^{\circ} \mathrm{C} \mathrm{min}^{-1}$.

chin in the blends, the single $T_{\mathrm{g}}$ of the blends shifts to higher temperature.

Although $i$-PHB and $s$-PHB have different stereochemical structures as mentioned above, they present some common characteristics when they are blended with catechin. Firstly, the crystallization of either $i$ PHB or $s$-PHB is restrained by the existence of catechin, which is expressed by the depression or disappearance of melting peaks of $i$-PHB and $s$-PHB. Furthermore, catechin keeps amorphous state in the blends when its content is less than $40 \%$. It is thought that catechin intermolecularly and strongly interacts with both $i$-PHB and $s$-PHB. Secondly, only one glass transition was observed both for $i$-PHB/catechin and $s$ $\mathrm{PHB} /$ catechin blends, which means that both $i$-PHB and $s$-PHB are miscible with catechin in the amorphous phase. Furthermore, $i$-PHB and $s$-PHB show almost the same $T_{\mathrm{g}}$ values in the pure state observed in the second DSC scan. Both the $i-\mathrm{PHB} /$ catechin and $s$ $\mathrm{PHB} /$ catechin blends show again quite similar $T_{\mathrm{g}}$ values, when the blend contains the same catechin content. Although $i$-PHB and $s$-PHB showed different physical properties either in the pure state or in the blends with catechin due to their different stereochemical structures, $i$ - and $s-\mathrm{PHB} /$ catechin blends had similar values of $T_{\mathrm{g}}$ when the component $i$ - or $s$-PHB is almost in the amorphous state.

\section{The $T_{\mathrm{g}}$ of the Blends Calculated by Gordon-Taylor Equation}

Over the years, numerous equations have been proposed to correlate the $T_{\mathrm{g}}$ of a miscible blend with its composition. These equations, including the Couchman equation, ${ }^{23}$ the Gordon-Taylor equation, ${ }^{24}$ the Fox 
equation, ${ }^{25}$ the Kwei equation, ${ }^{26}$ and so on, have been derived from the so-called free volume hypothesis or from thermodynamic arguments, assuming the continuity of the entropy of the mixture at $T_{\mathrm{g}}$.

The Couchman equation (eq 2) has been widely used to describe the relationship between the glass transition temperature of polymer blends and their composition, assuming that $\Delta C_{\mathrm{pi}}$ does not depend on the temperature.

$$
\begin{aligned}
\ln T_{\mathrm{g}}= & \left(W_{1} \Delta C_{\mathrm{p} 1} \ln T_{\mathrm{g} 1}+W_{2} \Delta C_{\mathrm{p} 2} \ln T_{\mathrm{g} 2}\right) /\left(W_{1} \Delta C_{\mathrm{p} 1}\right. \\
& \left.+W_{2} \Delta C_{\mathrm{p} 2}\right)
\end{aligned}
$$

where $T_{\mathrm{g}}$ is the glass transition temperatures of blend, $T_{\mathrm{gi}}$ is glass transition temperature of component $i, W_{\mathrm{i}}$ is the weight fraction of component $i$ and $\Delta C_{\mathrm{pi}}$ is the difference in specific heat for component $i$ between the liquid and glassy states at $T_{\mathrm{gi}}$.

This equation can be reduced to several other equations, following a certain number of simplifying assumptions. ${ }^{27}$ When $T_{\mathrm{g} 2} / T_{\mathrm{g} 1}$ is not very far from unity, then the logarithm expansion of eq 2 can be limited to the first term, after rearrangement, one finds the Gordon-Taylor equation (eq 3)

$$
T_{g}=\left(w_{1} T_{g 1}+k w_{2} T_{g 2}\right) /\left(w_{1}+k w_{2}\right)
$$

where $k=\Delta C_{\mathrm{p} 1} / \Delta C_{\mathrm{p} 2}$. The fitting constant $k$ is also thought to represent the ratio of the volume expansion coefficients of the components at the glass transition temperature or the ratio of the heat capacity of the components. $^{28}$

The application of the Gordon-Taylor equation to the $\mathrm{PHB} /$ catechin blend systems gives a good prediction of glass transition behavior by taking $k=0.9645$ for $i$-PHB/catechin blends and $k=0.8905$ for $s$ $\mathrm{PHB} /$ catechin blends as shown in Figure 5. Thus, both the blends are considered to be miscible systems in the amorphous phase.

\section{FT-IR Analysis of $s$-PHB/Catechin Blends and $i$ - PHB/Catechin Blends}

FT-IR spectroscopy is quite a suitable technique to investigate the intermolecular interaction like hydrogen-bonding. Usually, the changes of the strength and the position of IR absorption peaks resulting from some characteristic functional groups, like carbonyl and hydroxyl, can be attributed to the existence of intermolecular interaction. There are two main ranges in the infrared spectra that are sensitive to the formation of hydrogen bonds: one is the carbonyl vibration region in $1650-1800 \mathrm{~cm}^{-1}$ and the other is the hydroxyl vibration region in $3000-3600 \mathrm{~cm}^{-1}$.

In the systems investigated here, $i$-PHB and $s$-PHB with the same chemical structure have the carbonyl in

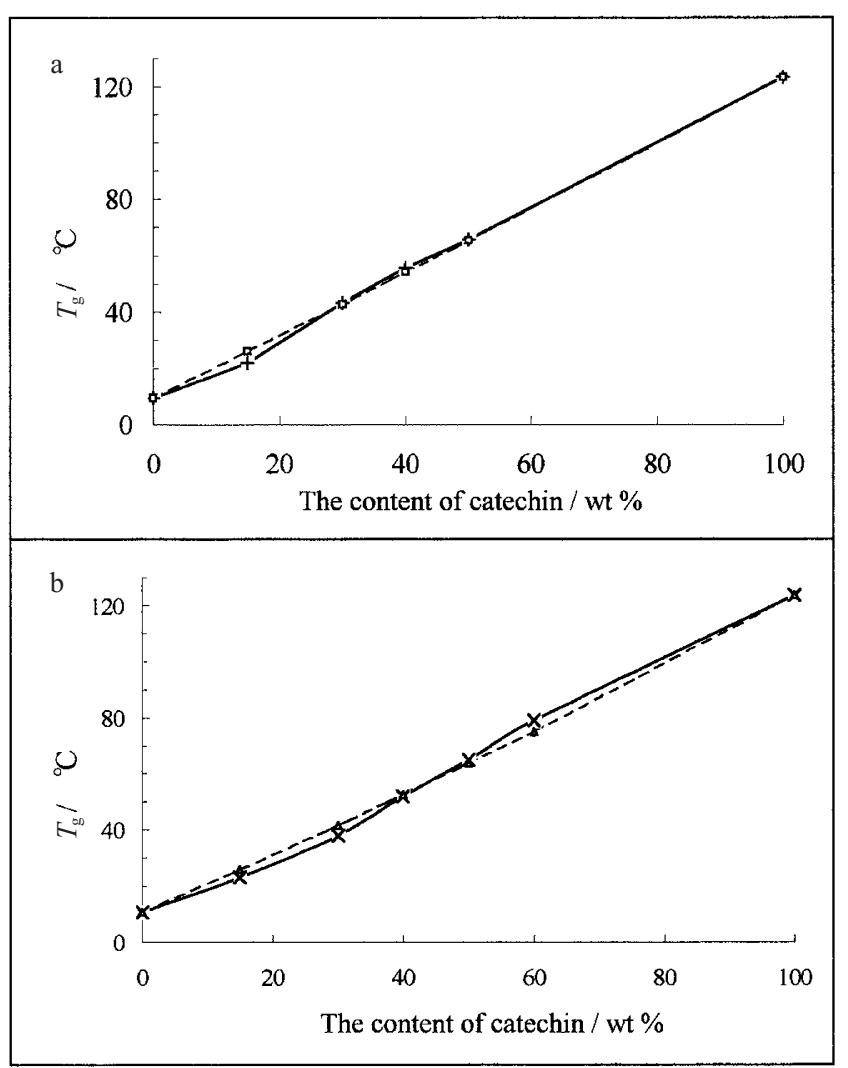

Figure 5. Experimental $T_{\mathrm{g}}$ and theoretically predicted $T_{\mathrm{g}}$ of the blends versus catechin weight fraction. (a): $i$-PHB/catechin blends; (b): $s$-PHB/catechin blends. (+): the $T_{\mathrm{g}}$ of $i$-PHB/catechin blends measured by DSC; ( $\square)$ : the $T_{\mathrm{g}}$ of $i-\mathrm{PHB} /$ catechin blends predicted by Gordon-Taylor equation with $k=0.9645 ;(\times)$ : the $T_{\mathrm{g}}$ of $s-\mathrm{PHB} /$ catechin blends measured by DSC; $(\triangle)$ : the $T_{\mathrm{g}}$ of $s$-PHB/catechin blends predicted by Gordon-Taylor equation with $k=0.8905$; solid line: experimental line; break line: GordonTaylor equation line.

the monomeric repeating unit that yields a $v_{\mathrm{C}=\mathrm{O}}$ stretching mode near $1724 \mathrm{~cm}^{-1}$, while catechin exhibits no absorption in the region from $1650-1800 \mathrm{~cm}^{-1}$. Therefore, any changes observed in the FT-IR spectrum in this region should be attributed to those in the chemical environment of carbonyls, such as the formation of hydrogen bonds.

In Figure 6 are shown the FT-IR spectra of $s$ $\mathrm{PHB} /$ catechin blends in the carbonyl vibration region (a) and in the hydroxyl vibration region (b), respectively, as a function of catechin composition. The carbonyl absorption peak of $s$-PHB is located at about $1739 \mathrm{~cm}^{-1}$, which shows $s$-PHB is an almost amorphous polymer, because the absorption in this high wavenumber is induced by the carbonyl in the amorphous component of PHB. ${ }^{16}$ When $s$-PHB is blended with catechin, not only the carbonyl absorption peak shifts to lower wavenumber but also a new peak located at $1713 \mathrm{~cm}^{-1}$ obviously appears especially when the content of catechin is more than $30 \%$, all of which 


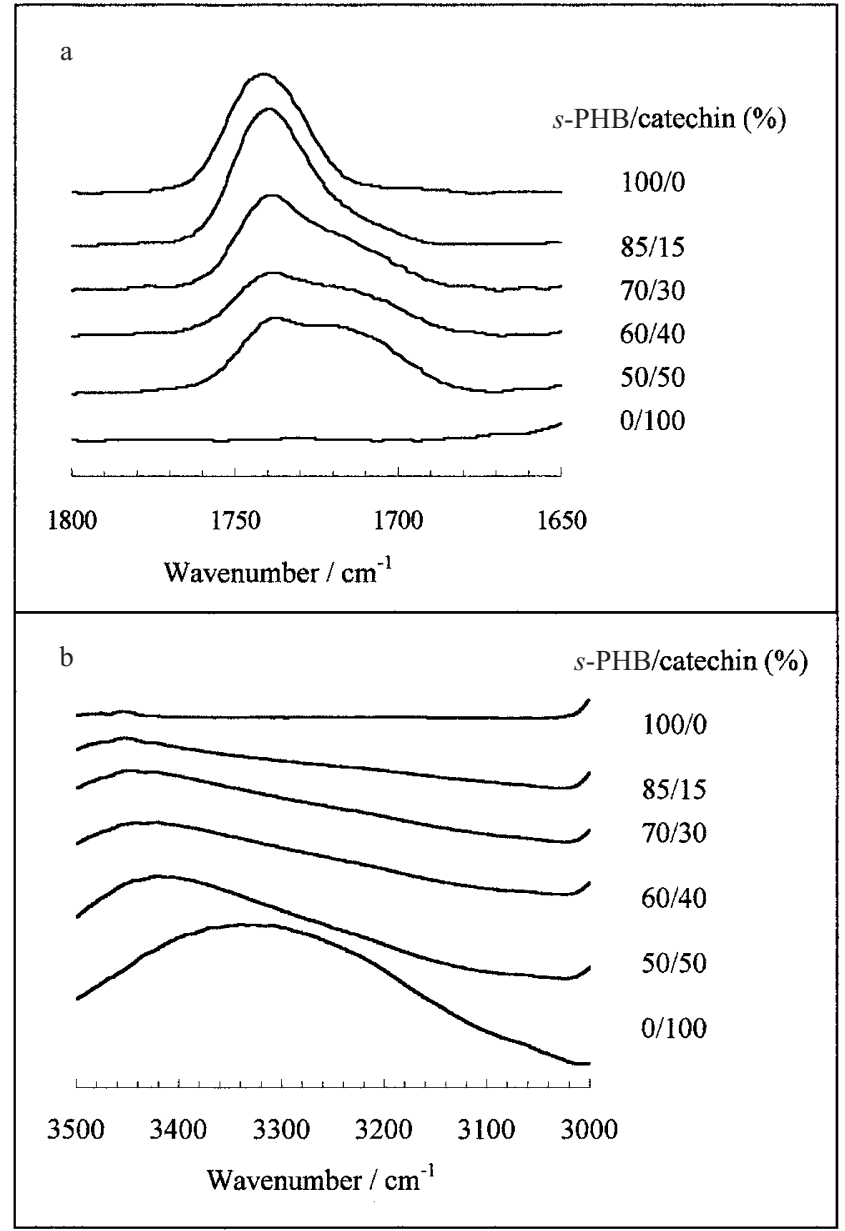

Figure 6. FT-IR spectra of $s$-PHB and its blends with various contents of catechin in the carbonyl vibration region (a) and in the hydroxyl vibration region (b).

are thought to be the characteristic for the formation of the intermolecular hydrogen bonds between $s$-PHB and catechin. $s$-PHB shows a weak absorption peak at $3450 \mathrm{~cm}^{-1}$, which should be attributed to the vibration of hydroxyl groups at the chain terminal of $s$-PHB.

Catechin has five hydroxyls in one molecule and its FT-IR spectrum shows one broad band centering at $3320 \mathrm{~cm}^{-1}$, at least a part of which should be assigned to the self-associated hydroxyl group because of the low wavenumber position. When catechin is blended with $s$-PHB, a new absorption peak appears at $3415 \mathrm{~cm}^{-1}$, which should be assigned to the catechin hydroxyl groups intermolecularly hydrogenbonded with the carbonyls of $s$-PHB. The difference between the wavenumber of self-associated hydroxyl groups $\left(3320 \mathrm{~cm}^{-1}\right)$ and that of intermolecular hydrogen-bonded one could be due to the balance between the number of broken catechin-OH...HOcatechin self-associations and the number of formed catechin-OH. . .O $=\mathrm{C}-s$-PHB bonds.

In Figure 7 are shown the FT-IR spectra of $i$ $\mathrm{PHB} /$ catechin blends in the carbonyl (a) and the hy-

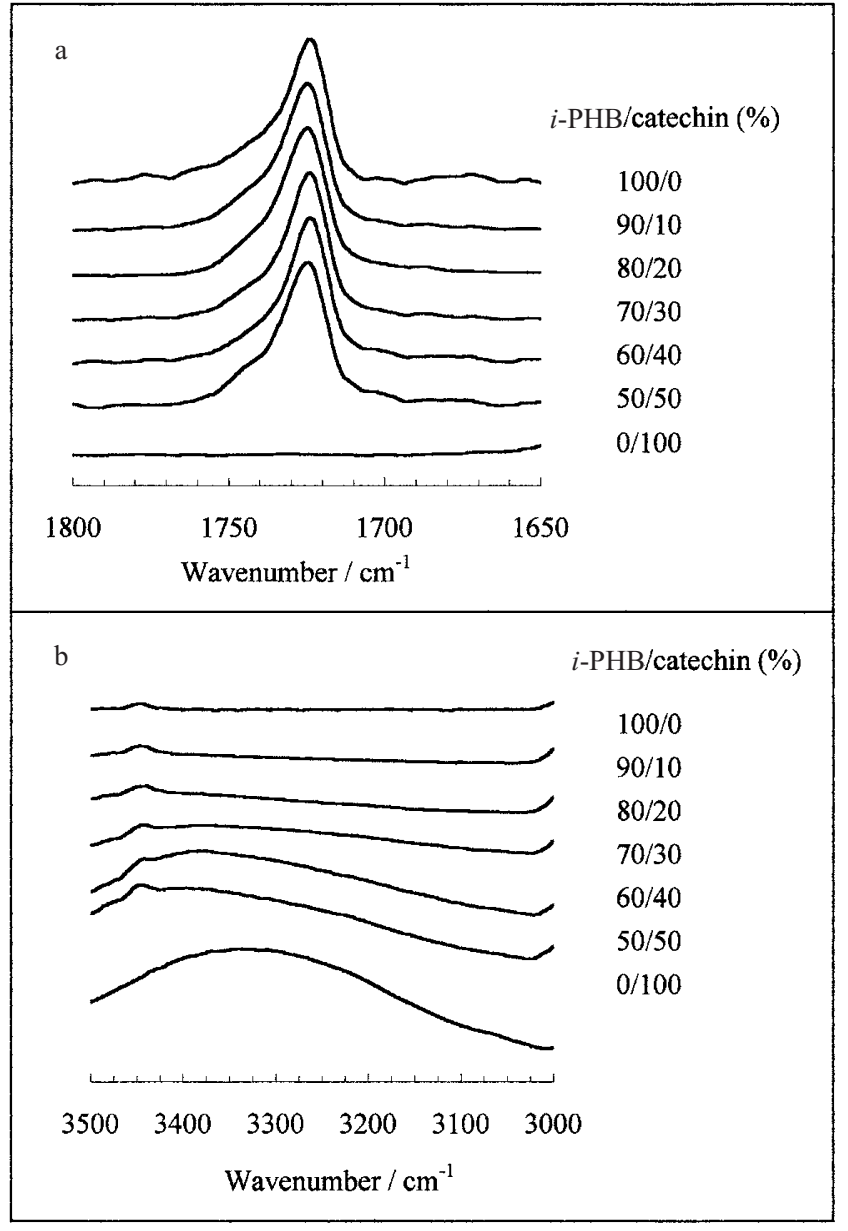

Figure 7. FT-IR spectra of $i$-PHB and its blends with various contents of catechin in the carbonyl vibration region (a) and in the hydroxyl vibration region (b).

droxyl vibration region (b) as a function of catechin composition. It is found that the absorption peak of carbonyl of the blends keeps almost at $1724 \mathrm{~cm}^{-1}$, but the absorption strength near $1739 \mathrm{~cm}^{-1}$ increases gradually. It is thought that the absorption in the higher wavenumber is induced by the amorphous part of $i$-PHB. $i$-PHB shows a weak absorption at about $3450 \mathrm{~cm}^{-1}$, which should be attributed to the vibration of hydroxyl groups at the chain terminal of $i$-PHB as described for $s$-PHB. When catechin is blended with $i$-PHB, a new absorption peak appears at $3375 \mathrm{~cm}^{-1}$, which should be again assigned to the catechin hydroxyl groups intermolecularly hydrogen-bonded with the carbonyl of $i$-PHB. However, intermolecular hydrogen bonds cannot be demonstrated by the absorption in the carbonyl region. It is concluded that not only the content but also the strength of intermolecular hydrogen bonds between $i$ PHB and catechin is low. $i$-PHB has a high ability to form crystalline phase even with high content of catechin, which is unfavorable to the formation of intermolecular hydrogen bonds.

The FT-IR experiment indicated that in the 
$\mathrm{PHB} /$ catechin blend systems there are three kinds of hydrogen bonds, i.e., catechin-OH...HO-catechin, catechin-OH. . O $=\mathrm{C}-s$-PHB and catechin-OH. . . $=\mathrm{C}-$ $i$-PHB. Usually, the stronger hydrogen-bonding is, the lower wavenumber in FT-IR spectra of hydroxyl groups is. Therefore, the strength of intermolecular hydrogen bonds increases as the order of $s-\mathrm{PHB} /$ catechin blends $<i$-PHB/catechin blends $<$ pure catechin.

\section{Curve-fitting Analysis of FT-IR Spectra}

Just as discussed above, the formation of the intermolecular hydrogen bonds between the ester carbonyl of $s$-PHB and the hydroxyl of catechin has been confirmed qualitatively by FT-IR. However, the intermolecular hydrogen bonds between the carbonyl of $i$ PHB and the hydroxyl of catechin are not shown obviously in the FT-IR spectra of the carbonyl absorption region. It is reasonably supposed that the almost all molecular chains of $s$-PHB are in the amorphous phase and they are easy to form hydrogen bonds with catechin but those of $i$-PHB are not so easy, because of high crystallinity. Here, the spectra in the carbonyl region are analyzed with a curve-fitting program based on the Gauss-Newton iteration procedure.

In Figure 6a, the FT-IR spectrum of $s-\mathrm{PHB} /$ catechin $(60 / 40)$ in the carbonyl region recorded at room temperature exhibits two components approximately at 1739 and $1713 \mathrm{~cm}^{-1}$, which should be reasonably attributed to $s$-PHB components in the hydrogen bond free amorphous and hydrogen-bonded amorphous state, respectively. The component corresponding to the crystalline state is not found since $s$-PHB is in the amorphous state in the $s-\mathrm{PHB} /$ catechin $(60 / 40)$ blend as shown by DSC. When a curve-fitting program is employed to quantitatively analyze the spectrum regarding the integrated intensity of these two separated bands, the experimental spectrum has been divided into two regions, that is, the amorphous and hydrogen-bonded components as shown in Figure 8a. The excellent agreement with error only about $3 \%$ between the experimental and fitted spectra indicates the reliability of this fitting technique.

In Figure 7a, the FT-IR spectrum of $i$-PHB/catechin $(60 / 40)$ in the carbonyl region recorded at room temperature exhibits only two components approximately at 1739 and $1724 \mathrm{~cm}^{-1}$, which should be attributed to $i$ PHB in the amorphous and crystalline components, respectively. The component corresponding to hydrogenbonding is not found. In Figure $8 \mathrm{~b}$, the experimental and fitted spectra for the $i$-PHB/catechin $(60 / 40)$ blend in the carbonyl vibration region are illustrated. The experimental spectrum has been divided into two peaks for the amorphous and the crystalline compositions. When the $i-\mathrm{PHB} /$ catechin $(60 / 40)$ sample is

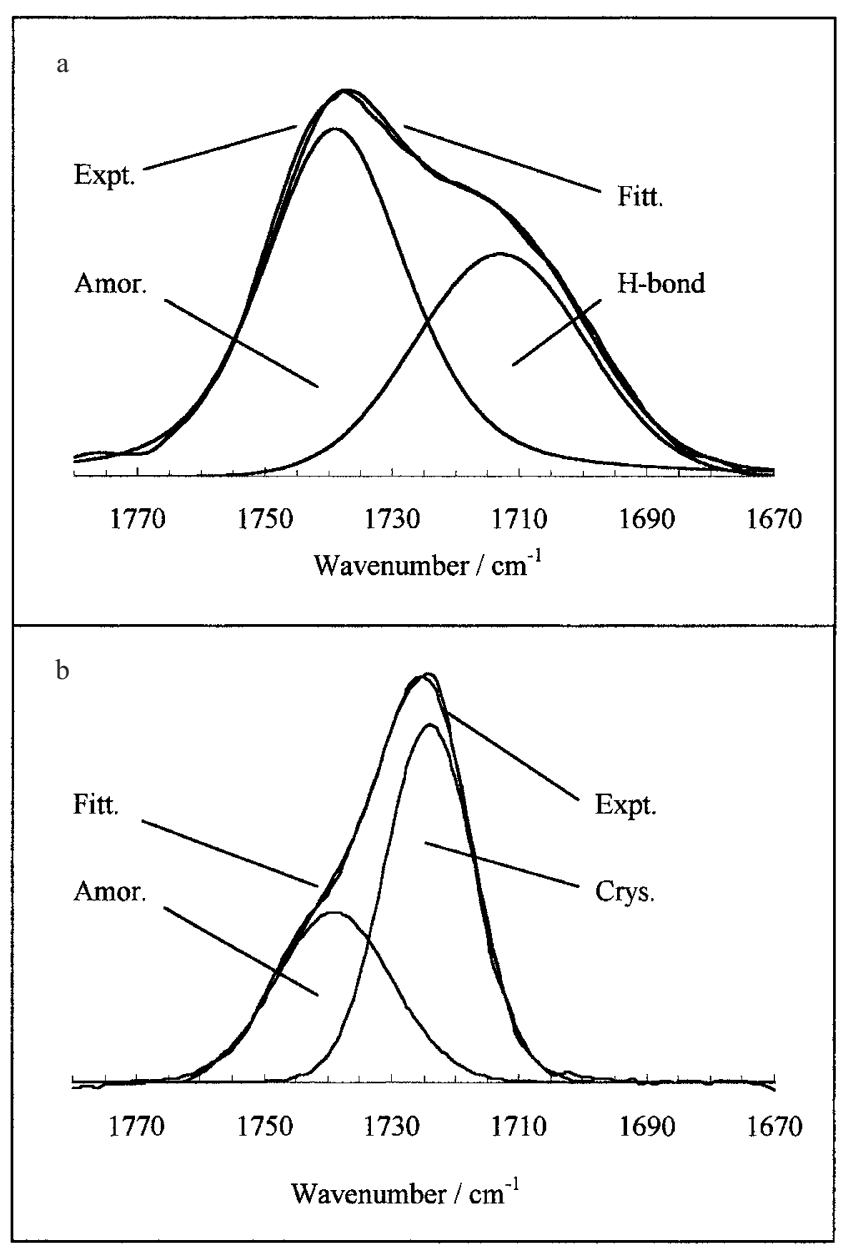

Figure 8. Experimental and fitted FT-IR spectra for the $s$ $\mathrm{PHB} /$ catechin (60/40) (a) and $i$-PHB/catechin (60/40) (b) blends in the carbonyl vibration region recorded at room temperature. Expt.: experimental spectrum; Amor.: amorphous component; Crys.: crystalline component; H-bond.: hydrogen-bonded component; Fitt.: fitted spectrum, i.e., the sum of the amorphous component and hydrogen-bonded component.

heated to $190^{\circ} \mathrm{C}$ and kept $5 \mathrm{~min}$, at which temperature the crystal phase of $i$-PHB disappears, and then the sample is scanned by FT-IR again. As shown in Figure 9, it is found that the FT-IR spectrum also exhibits two components, but they are approximately at 1739 and $1713 \mathrm{~cm}^{-1}$, which are just the same wavenumbers found for $s$-PHB/catechin (60/40) blend [Figure 6a] and so they should be attributed to $i$-PHB in the amorphous and hydrogen-bonded components, respectively. The component corresponding to the crystalline phase is not found. When the curve-fitting program is employed to quantitatively analyze the spectrum, it also shows the excellent agreement between the experimental and fitted spectra as shown in Figure 9.

The intermolecular hydrogen-bonding is not obviously observed in the carbonyl region in the FT-IR spectrum for $i$-PHB/catechin (60/40) at room temperature, but it shows strong intermolecular hydrogen- 


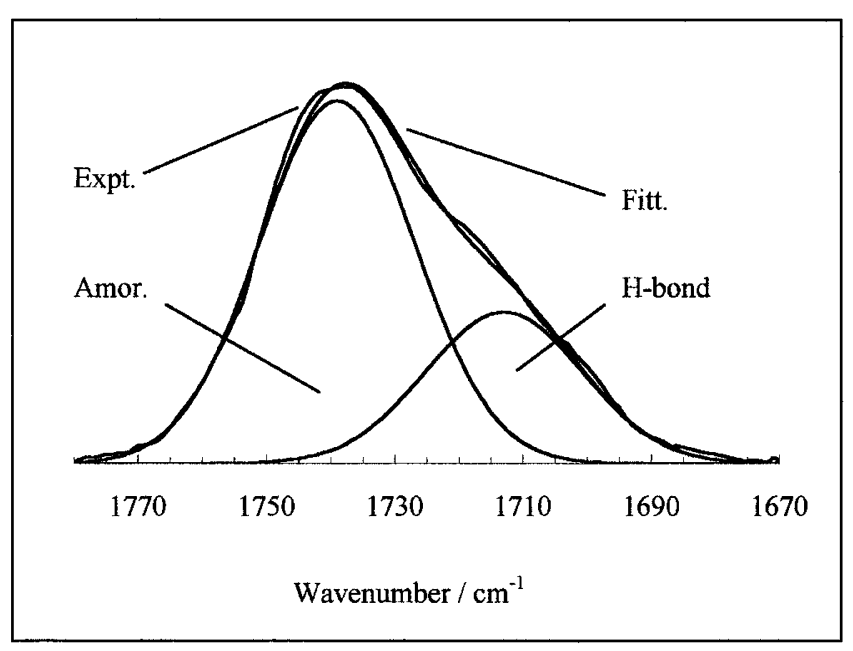

Figure 9. Experimental and fitted FT-IR spectra for the $i$ $\mathrm{PHB} /$ catechin $(60 / 40)$ blend in the carbonyl vibration region recorded at $190^{\circ} \mathrm{C}$. Expt.: experimental spectrum; Amor.: amorphous component; H-bond.: hydrogen-bonded component; Fitt.: fitted spectrum, i.e., the sum of the amorphous component and hydrogen-bonded component.

bonding at $190^{\circ} \mathrm{C}$ when all the $i$-PHB component is in the amorphous state. According to the quantitative analysis on the carbonyl region for $s$ - and $i$ $\mathrm{PHB} /$ catechin $(60 / 40)$ blends, it is concluded that the existence of crystal phase of $i$-PHB is unfavorable to form the hydrogen bonds between $i$-PHB and catechin. The DSC result, that $i$-PHB and $s$-PHB have no great difference when they are in the amorphous state even though they have different stereochemical structures, is confirmed again by FT-IR.

\section{CONCLUSIONS}

The thermal properties including crystallinity, $T_{\mathrm{m}}$ and $T_{\mathrm{g}}$ of $i$-PHB and $s$-PHB were greatly modified by the addition of catechin. Furthermore, it was found that both $i$-PHB and $s$-PHB were miscible with catechin in the amorphous phase. Although $i$-PHB and $s$ PHB showed different physical properties either in the pure state or in the blends with catechin due to their different stereochemical structures, $i$ - and $s$-PHB/catechin blends had similar values of $T_{\mathrm{g}}$ when the component $i$ or $s$-PHB was almost in the amorphous state.

Not only the content but also the strength of intermolecular hydrogen-bonding between $i$-PHB and catechin is low due to the high crystallizability of $i$-PHB. However, the FT-IR spectra of $s$-PHB/catechin blends showed obvious characteristic for the intermolecular hydrogen bonds. By comparing the hydroxyl region for catechin and its blends with PHB, it is concluded that the strength of intermolecular hydrogen bonds is in the order: $s$-PHB/catechin blends $<i$-PHB/catechin blends
$<$ pure catechin.

By applying a curve-fitting program for FT-IR spectra in the carbonyl region, the spectra of $s$ $\mathrm{PHB} /$ catechin $(60 / 40)$ and $i$-PHB/catechin $(60 / 40)$ at room temperature and at $190^{\circ} \mathrm{C}$ have been divided and analyzed. It was concluded that the existence of crystal phase of $i$-PHB was unfavorable for the formation of hydrogen bonds between $i$-PHB and catechin at room temperature.

The formation of intermolecular hydrogen bonds between PHB and catechin has been confirmed. The properties of PHB can be greatly modified by the addition of catechin. The results indicate that it is acceptable to use naturally produced polyphenol to modify proton-acceptor polymers like polyesters. The effect of hydrogen-bonding on the properties like biodegradability and crystallization of PHB is in progress now.

\section{REFERENCES}

1. P. Xing, L. Dong, Y. An, Z. Feng, M. Avella, and E. Martuscelli, Macromolecules, 30, 2726 (1997).

2. D. Li and J. Brisson, Macromolecules, 29, 868 (1996).

3. X. Zhang, K. Takegoshi, and K. Hikichi, Macromolecules, 26, 2198 (1993).

4. J. Li, Y. He, and Y. Inoue, Polym. J., 33, 773 (2001).

5. Y. He, N. Asakawa, and Y. Inoue, Macromol. Chem. Phys., 202, 1035 (2001).

6. Y. He, N. Asakawa, J. Li, and Y. Inoue, J. Appl. Polym. Sci., 82, 640 (2001).

7. Y. He, N. Asakawa, and Y. Inoue, J. Polym. Sci. Part B: Polym. Phys., 38, 2891 (2000).

8. P. Pedrosa, J. A. Pomposo, E. Calahorra, and M. Cortázar, Polymer, 36, 3889 (1995).

9. M. Friedman and H. S. Jürgens, J. Agric. Food. Chem., 48, 2101 (2000).

10. J. J. Dalluge and B. C. Nelson, J. Chromatogr. A, 881, 411 (2000).

11. T. G. Toschi, A. Bordoni, S. Hrelia, A. Bendini, G. Lercker, and P. L. Biagi, J. Agric. Food. Chem., 48, 3973 (2000).

12. K. E. Uhrich, S. M. Cannizzaro, R. S. Langer, and K. M. Shakesheff, Chem. Rev., 99, 3181 (1999).

13. Y. Ikada and H. Tsuji, Macromol. Rapid. Commun., 21, 117 (2000).

14. H. Abe, I. Matsubara, and Y. Doi, Macromolecules, 28, 844 (1995).

15. Y. Inoue and N. Yoshie, Prog. Polym. Sci., 17, 571 (1992).

16. Y. He, J. Li, X. Shuai, and Y. Inoue, Macromolecules, 34, 8166 (2001).

17. Y. Horii and T. Hagiwara, Int. J. Biol. Macromol., 25, 237 (1999).

18. Y. He and Y. Inoue, Polym. Int., 49, 623 (2000).

19. P. Barham, A. J. Keller, E. L. Otun, and P. A. Holmes, J. Mater. Sci., 19, 2781 (1984).

20. M. Aubin, and R. E. Prud'homme, Macromolecules, 21, 10 (1988). 
21. G. Bisso, P. Casarino, and E. Pedemonte, Thermochimica Acta, 321, 81 (1998).

22. S. W. Kuo, C. F. Huang, and F. C. Chang, J. Polym. Sci. Part B: Polym. Phys., 39, 1348 (2001).

23. P. R. Couchman, Macromolecules, 11, 1156 (1978).

24. M. Gordon and J. S. Taylor, J. Appl. Chem., 2, 493 (1952).
25. T. G. Fox, Bull. Am. Phys. Soc., 1, 123 (1956).

26. T. K. Kwei, J. Polym. Sci., Polym. Lett. Ed., 22, 307 (1984).

27. M. Aubin and R. E. Prud'homme, Macromolecules, 21, 2945 (1988).

28. A. A. Lin, T. K. Kwei, and A. Reiser, Macromolecules, 22, 4112 (1989). 